\author{
Military Technical College \\ Kobry El-Kobbah, \\ Cairo, Egypt
}

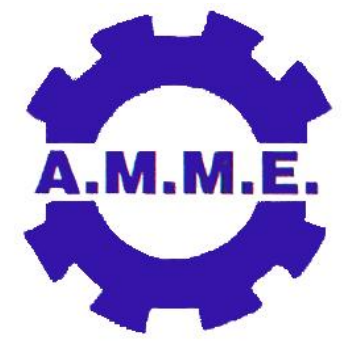

14th International Conference on

Applied Mechanics and

Mechanical Engineering.

\title{
Laser-induced photoacoustic imaging for breast cancer detection using multivariate image analysis.
}

\author{
By \\ Yasser H. Elsharkawy ${ }^{1} \quad$ W.M. Hussein ${ }^{2} \quad$ Mohamed Abbass $^{3}$
}

\begin{abstract}
:
Time-resolved photoacoustic imaging has been used to characterize breast tissue for the purpose of discriminating between normal and maligned areas of the tissue. Ultrasonic thermoelastic waves were generated in breast tissue by the absorption of nanosecond laser pulses at $193 \mathrm{~nm}$ produced by Q-switched Excimer laser in conjunction with a Michelson interferometer used to detect the thermoelastic waves and plot the 2-D and the 3-D image through IR- CCD. The concepts behind the use of photo-acoustic techniques for off-line detection of breast cancer tumor features were presented in earlier research papers [1][2]. This paper illustrates the application of multivariate image analysis (MIA) techniques to detect the presence of tumor features of breast cancer. MIA is used to rapidly detect the presence and quantity of common tumor features as they scanned by an RGB camera. Multiway principal component analysis is used to decompose the acquired three-channel tumor images into a two dimensional principal components (PC) space. Masking score point clusters in the score space and highlighting corresponding pixels in the image space of the two dominant PCs enables isolation of tumor defect pixels based on contrast and color information. The technique provides a qualitative result that can be used for early tumor detection. The proposed technique can potentially be used on-line to prescreen the existence of tumors through vision based systems.
\end{abstract}

Keywords: Mul Photoacoustic imaging, Characterizing, Breast, Tumor detection, Multivariate image analysis 


\section{* Egyptian Armed Forces, Cairo, Egypt \\ 1-Introduction}

Recent studies indicate that breast cancer affects one of every eight women in the unit states (American Cancer Society 1991)[17]. Early detection can greatly improve a woman's chances for survival. It is estimated that approximately $20 \%$ of breast cancers are missed in conventual's mammography screening. Several risk factors for breast cancer have been identified.

\section{1-1 Physiology of malignant breast masses:}

It is believed that cancer cells in the breast stimulate the growth of fibrous tissues. This pattern of growth, termed thermoplastic reaction, is what gives malignant breast masses a dense or hard consistency. Carcinoma cells grow in the path of least resistance. When the surrounding tissues are firm and have a mostly glandular constituency, as found in younger women, the tumor cells tend to grow in clefts between fibrous regions. When the surrounding tissues are soft and have are soft and have a more fatty consistency, tumor cells grow in all directions. Many primary malignancies exhibit a satellite (star-shaped) pattern or speculated appearance. The degree of desmoplastic reaction or deformation of surrounding tissues due to bonding between the tumor and connective tissues will alter the tumor and connective tissue response to palpation. Thermoelstic deformation measurement of local tissue provides a means of quantifying tumor and connective tissue response to palpation. This information could be potentially used in the early detection and classification of many developing breast cancer.

In this paper we used the photoacoustic imaging techniques for visualizing the internal structure of soft biological tissues is currently exciting much interest. This approach to medical imaging relies upon low energy, sub-ablation threshold nanosecond laser pulses are absorbed in the tissue, producing ultrasonic thermoelastic waves. The amplitude and temporal characteristics of the thermoelastic waves depend strongly upon the optical properties of the tissue and it has been suggested that, by exploiting the preferential optical attenuation in abnormal tissues, the photoacoustic signature could be used to identify tumor location for breast tissue. In addition, the reflection and generation of subsurface thermoelastic waves can provide information about the structure and thickness of it.

The photoacoustic signals are then non-contact detected and spatially resolved to reconstruct a 3D image of the internal tissue structure in much the same way that volumetric images. A key advantage of the technique is that it exploits the strong optical contrast of tissues enabling differentiation of anatomical features that would be indistinguishable using other radiological modalities. A broad range of potential applications have emerged including detection of breast [3][4], skin and oral cancers3

The aim of the work described in this paper was to exploit the increased optical absorption in maligned tissue, reported by others for discrimination purposes. For this reason, laser pulses at $308 \mathrm{~nm}$ were used to generate photoacoustic signatures in tumor tissue. These signatures were actually multivariate images of the maligned tissue.

Interpretation of multivariate images is done in a two stage procedure. After preprocessing the images, the content is visualized and analyzed through direct visual inspection. In a 
subsequent step, quantitative data are extracted from the images and analyzed applying data mining methods. Many algorithms have been proposed in the fields of statistics, pattern recognition, machine learning $(\mathrm{ML})$ and artificial neural networks (ANN). Although these techniques have been applied in numerous engineering and data mining applications, applications to biomedical imaging domains are still reported rather exceptional [18]. One of the most prominent techniques is the Principal Component Analysis (PCA) [19], which facilitates a projection from a higher dimensional signal space into a lower dimensional space according to the data variance. Multivariate image analysis (MIA) [20-24] involves the use of multiway PCA to decompose three-dimensional images and collect features in the image or scene space that have similar spectral features into common regions in the PCA score space. It is this spatial independence of PCA that makes MIA powerful at analyzing images for spectrally similar features.

This paper illustrates the application of multivariate image analysis (MIA) techniques to detect the presence of tumor signatures of breast cancer. MIA is used to rapidly detect the presence and quantity of common tumor signatures as they scanned by an RGB camera. Multiway principal component analysis is used to decompose the acquired three-channel tumor images into a two dimensional principal component (PC) space.

The paper is structured as follows. Some imaging system details are presented in section2. Section 3 reviews the main concepts of MIA using the multiway PCA. This is followed by an off-line MIA technique through an example of tumor detection from a human sample image.

\section{1-2 Review of multivariate Image Analysis}

This paper assumes that the reader has a basic understanding of PCA as it is applicable to MIA [25]. This section provides a brief review of PCA and MIA and illustrates their use with an off-line study of tumor identification from an RGB image of one tumor sample.

MIA techniques, first introduced by Esbensen et al. [26], consist of extracting feature information from multivariate images using Multiway PCA (MPCA). A multivariate image consists of a group of matched images, with each image in the group representing a unique variable. Such an image can be represented as a three dimensional data set , where first two dimensions represent pixels in the image plane and the third dimension represents the variable index. For example, a low resolution image from a digital camera typically consists of 640 by 480 pixels over three colour channels, forming a three dimensional array. The entries in this array are integers between 0 and 255 .

These images are often referred to as RGB images, corresponding to the red, green and blue channels. Converting this 3-D array to a two-dimensional matrix is termed "unfolding" or "reshaping". There are six possible ways to unfold a 3-way array into a 2-D matrix. We slice along each dimension of the 3-way array and place these slices next to each sideby-side or top-to-bottom. Only two of these reshaping operations are useful for image processing and are illustrated in Figure 1 . The result of either method is that one obtains a matrix $X$ that has $1 \times J$ rows with $K$ columns. Simply speaking, the first case moves rowwise across the image, the second case moves column-wise up and down the image. It does not matter how we unfold the image matrix, as long as each pixel in the image has a unique row assigned to it in the unfolded matrix. We also need to do this in reverse order; we want to be able to refold the matrix back up into an image. Once we have a two-way 
array or matrix $\mathrm{X}$ we can carry out principal component analysis. This matrix $\mathrm{X}$ will have $\mathrm{N}$ $=\mathrm{I} \times \mathrm{J}$ rows and $\mathrm{K}$ columns, implying that XTX is only a $3 \times 3$ matrix and the full loading matrix, $P$ is also a $3 \times 3$ matrix for an RGB image. The full score matrix $T$ has dimensions $(\mathrm{I} \cdot \mathrm{J}) \times \mathrm{K}$, and performing our unfolding operation in reverse order allows us to obtain $\mathrm{K}$ matrices of size $\mathrm{I} \times \mathrm{J}$, the dimensions of our original image $\mathrm{X}$. So performing PCA on an image is equivalent to decomposing the image into K successive "score images", the first one of which contains most of the original information, with a decreasing amount in the remaining score images.

We also saw that we can obtain residuals matrices, $\mathrm{Ei}$, after extracting successive principal components. Each of these residual matrices show how far away from the PCA model we are built it. All the analyses were coded using MACCMIA software developed by McMaster University Control Consortium, Hamilton, Ontario, Canada.

\section{1-3 Description of photoacoustic signal generation.}

As first proposed by Callis et al. [1], pressure pulses in the illuminated sample arise from the volume changes produced by radiationless relaxation ( $\Delta V$ th $)$ and structural rearrangements at the molecular level $(\Delta V \mathbf{r})$. Relaxation originates either from nonradiative decay of excited states or heat release (enthalpy change) in photoinitiated reactions, including the heat involved in the rearrangement of the sample. The structural volume changes reflect movements of the photoexcited molecules and/or the surrounding tissue in response to such events as dipole moment change, charge transfer, and photoisomerization.

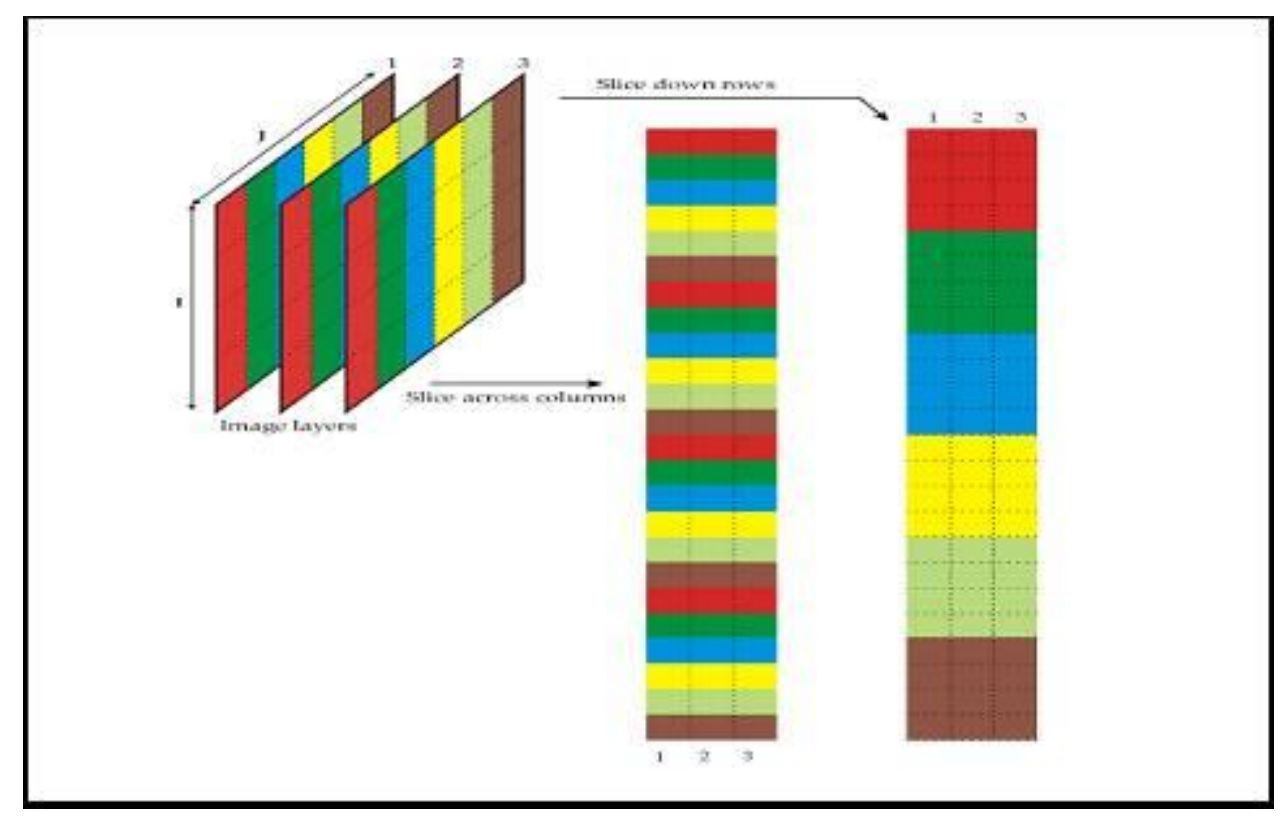

Figure1. Converting a 3-D image structure to a 2-D matrix by unfolding [27]

A theoretical model based on the theory of elasticity was developed to describe the time dependent deformation of a thin polymer, following absorption of a short laser pulse. A numerical time dependent solution of the thermoelastic wave equation has been obtained for laser-induced heating of a polymer film in a three-dimensional cylindrical symmetric geometry. This solution shows that the sample will undergo thermelastic expansion,[5][6][7]as the surface will reach a new equilibrium position. The time constant 
of this expansion is governed by the ratio of the 1/e depth of laser light absorption, to the speed of the sound in the sample. $1 / \mu_{\text {eff }} \mathrm{C}$, and the thermal relaxation time is $\mathrm{a} / \mathrm{k} \mu^{2}{ }_{\text {eff }}$, where $\mu_{\text {eff }}$ is the effective attenuation coefficient, $C$ the longitudinal sound speed, $k$ the thermal diffusivity and a numerical constant depend on geometry.

The initial light distribution can be approximated by an exponentially decaying profile with tissue depth $(z>0)$, and the radial profile $L(r)$. The laser profile is

$$
I(r, z) \cong I_{0} L(r) \exp \left(-\mu_{\text {eff }} z\right) \text {. }
$$

In the turbid medium such as tissue, light is subjected to both absorption and scattering.The three-dimensional temperature distribution can be approximated by

$$
T(r, z) \cong T 0 \exp (-\mu \text { eff } z) \text { and } T 0=\Phi \mu \mathrm{a} / \rho C v
$$

where $\mathrm{Cv}$ is the heat capacity (constant volume), $\Phi$ is laser fluency (energy per unit area) and $\rho$ is mass density. The non-zero temperature distribution results in internal stresses that lead to thermoelastic deformation. This deformation in a solid body is determined by the thermoelastic wave equation [8]

$$
\rho \partial 2 \mathrm{u} / \partial \mathrm{t} 2-\mathrm{E} \nabla 2 \mathrm{u} / 2(1+\mathrm{v})-\mathrm{E} \nabla(\nabla \cdot \mathrm{u}) / 2(1+\mathrm{v})(1-2 \mathrm{v}) \quad=-\mathrm{E} \beta \nabla \mathrm{T} / 3(1-2 \mathrm{v})
$$

Subject to the appropriate initial and boundary conditions. $\mathrm{u}$ is the displacement Vector, $\mathrm{E}$ is Youn's modulus; $v$ is Poisson's, $\beta$ is the thermal expansion and $T$ is the laser-induced temperature increase above a uniform ambient level. The photoacoustic method detects the time dependent heat generated in a sample via interaction with pulsed or intensity modulated optical radiation. Such interaction also induces a number of thermal and thermoelastic effect in a sample. In particular it causes the generation of surface and bulk acoustic waves. The detection of acoustic waves is the basis of photoacoustic methods. The informative features of this method allow one to determine sample thermal, optical, and acoustical properties that depend on peculiarities of sample structure [9].

We design and implement an optical interferometer sensor that cam measure timeresolved photoacoustic and photothermal response simultaneously. The sensor has great diagnostic power since it would be able to measure the optical, thermal, acoustical and physical properties of the tissue. The calibration was extrapolated to an extremely low intensity range where displacements of the order of magnitude of $10^{-14} \mathrm{~m}$. The system sensitivity is $0.951 \mu \mathrm{v} / \mathrm{pa}$ over $35 \mathrm{MHz}$ measurements. Coupled charge device (CCD) camera used to reconstruct the 3-D image for acoustic-induce surface displacements in the heated volume explaining the thermal response for the sample providing advantage in term of the spatial sampling, smaller element sizes and spacing.

The system consist of a designed optical interferometric technique was used to measure laser-induced surface expansion, photoacoustic, photothermal of a sample with a spatial resolution of approximately $4 \mathrm{~nm}$ and temporal resolution of $3 \mathrm{~ns}$. Q-switched excimer laser at a wavelength $193 \mathrm{~nm}$ and pulse duration $8 \mathrm{~ns}$ provides the pump laser pulse. The beam is focused to $8 \mathrm{~mm}$ diameter spot on thin polymer film $(1 \mu \mathrm{m})$ placed on the sample surface as shown in Figure 2. The interferometric probe light from a cw He-Ne laser. A 50/50 beamsplitter is used in a Michelson interferometer set-up. A $5 \mathrm{~cm}$ lens focuses the light onto the sample through the polymer film. The sample motion modulates the frequency of the fringe pattern as shown in Figure 1(b), and one can determine the sample surface 
displacements. Due to the thermal diffusion the refractive index of thin polymer film has changed after thermal relaxation occur. The light is combined and directed towards photodiode and also to charge coupled device IR-CCD camera after passing through HeNe wavelength filter to remove the probe beam from the reconstructed image. The photodiode signal is captured and digitized at a rate of 1 giga sample/sec by a digital oscilloscope and transferred to a computer for analysis.

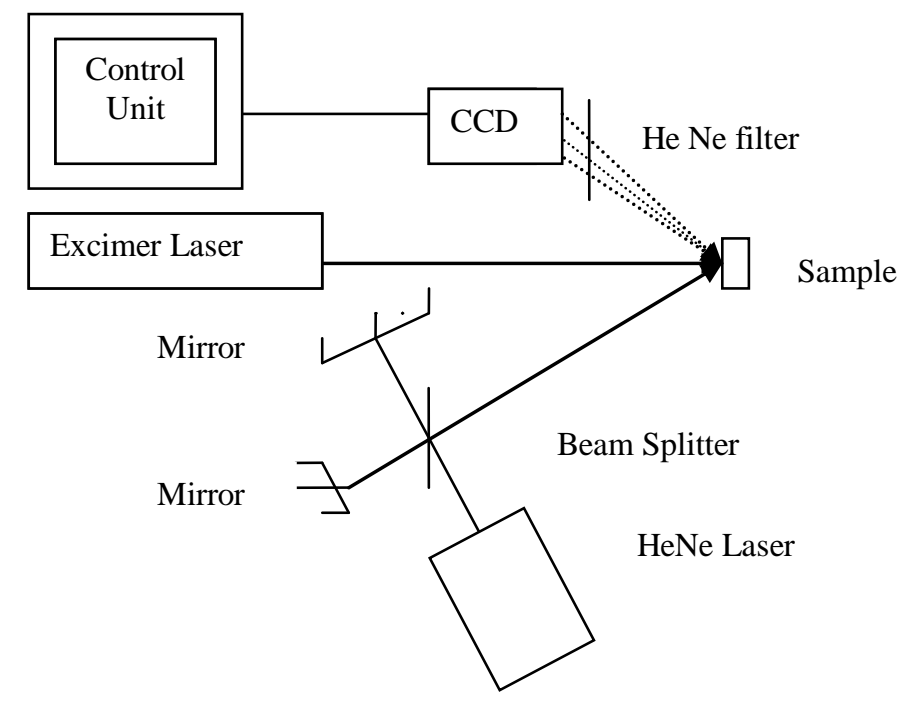

(a)

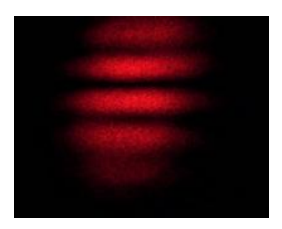

Fringe mode

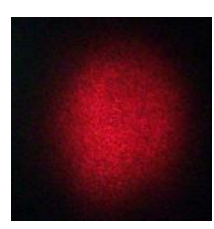

Speckle due to fringe modulation

(b)

Figure 2. (a) block diagram of the photoacoustic imaging backward mode, (b) The Fringe and Fringe modulation images results from sample surface displacements

The time-resolved imagings for diagnostic measurements of thermoelastic wave evaluation in the samples were based on Michelson interferometer shown in Figure 2. The recorded images the thermoelastic wave's propagation about $1 \mu$ s after laser irradiation the actual dimension of each scene was about $5 \mathrm{~mm} \times 5 \mathrm{~mm}$.

Recorded images at time delays greater than 1 ps (as shown in Figure 2), when the surface expansion is already occurred, provide information on photoacoustic processes induced by laser. Images are more complex, being composed of a main dark ring, corresponding to the expanding front of the hemispherical thermoelastic wave, and two sets of fine fringes located internally and externally with respect to this dark ring. Figure 3 illustrates how these fringe structures originate. Below the diagram of the thermelastic wave, we sketched a sample radial profile of the density distribution that can be reasonably associated with the observed thermoelastic event, according to theoretical models of thermoelastic wave dynamics [8]. It is composed of the front of the traveling thermoelastic wave. In a very schematic manner, it can be assumed that the interference between undisturbed probe rays passing out of the thermoelastic wave and those deflected by the thermoelastic rear produces the internal fine fringes, while the external 
ones are due to the interference between the slightly perturbed rays crossing the saddle behind the density peak and those deflected by the thermoelastic leading front. Assuming for simplicity that deflected and undisturbed rays give rise to two distinct focal rings (points $F_{t}$ and $F_{r}$, in Figure 3) generated by the thermoelastic front and the thermoelastic rear, respectively, the distance between each ring and the observation plane can be calculated from the width of the larger fringe of each distribution, according to the relationship $\mathrm{L}_{\mathrm{f}, \mathrm{r}}=$ $\mathrm{d}_{\mathrm{f}, \mathrm{r}}{ }^{2} / 2 \lambda[10]$. This provides information about the minimum distance where the collecting camera can be positioned. Also worth noting is that the dark ring separating the two sets of fringes is not a zero of the interference pattern, but a shadowgraph corresponding to the peak of the density distribution. As a consequence, the radius of this ring does not depend on the distance between the ablation region and the observation plane, permitting direct measurements of the thermoelastric radius $r$, without scaling factors. In our experimental conditions we verified this behavior on values of $L$ varying from 3 to $30 \mathrm{~cm}$. In diffraction patterns of spherical thermoelastic, another more internal fringe structure is typically observed (and the so-called 'grey ring'), due to probe ray deflections on the border of the central vacuum region of the density distribution.

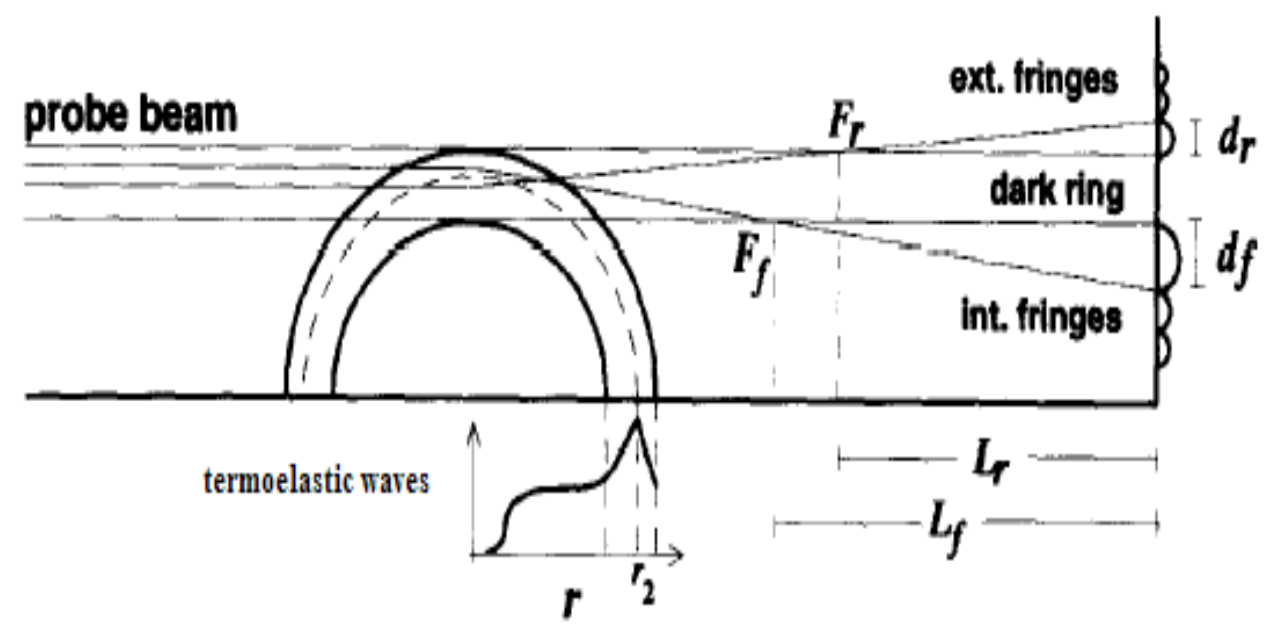

Figure 3. Interpretation of the fringe pattern generated by the probe beam crossing a thermoelastic waves

\section{3- Results}

The above technique is illustrated by the following RGB image of a specimen of a breast tissue containing tumor traces. Figure (4) illustrates the RGB multivariate image of size $146 \times 305 \times 3$ pixels of the tumor sample. The image depicts the normal tissue with the tumor traces. MPCA is used to decompose the three channel tumor image into two principal components. Feature information extracted by the two PCs and the residual, can be visually determined by observing the refolded score matrices T1 and T2 and the residual matrix $E$ as individual gray-scale images, as shown in figure (4). It can be seen from the T1 image that the first principal component extracts contrast information between normal tissue (the main specimen with bright pixels) and tumor traces (the red traces in the middle of the specimen). The T2 and the residual didn't reveal any information. The cumulative percent sum of squares in the multivariate image as explained by the first two PCs is $99.871 \%$ (94.12 and $5.751 \%$ respectively). As a result, only $A=2$ principal components are used in the analysis.

The loading vectors for these two dimensions are [red green blue] $P_{1}^{T}=[-0.575-0.545-0.61]$ and $P_{2}^{T}=[-0.089-0.700 .708]$. From these loading values, it can be seen that the first PC represents almost an average of the three colors [22], 
whereas the second PC represents mainly a contrast between green and blue colors. This contrast was triggered from the use of optical filter (He-Ne filter).

RGB Image

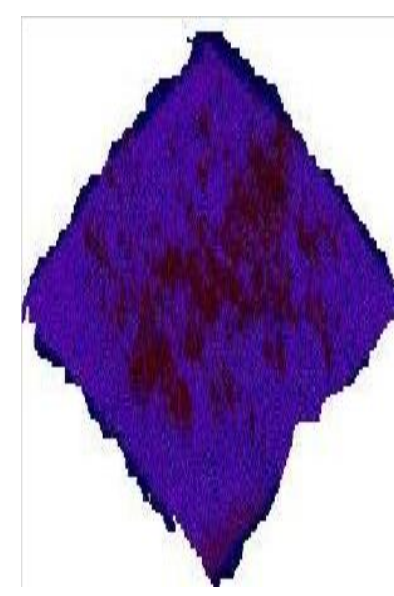

T1 Image

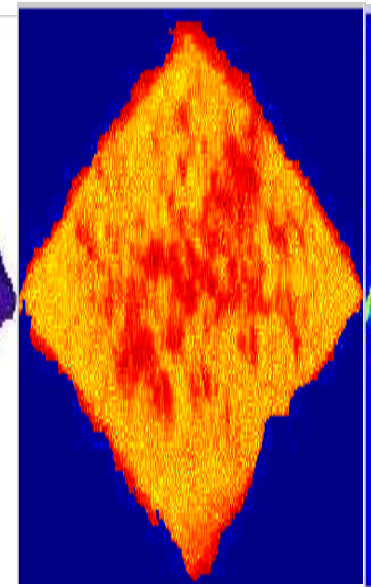

T2 Image Residual Image E

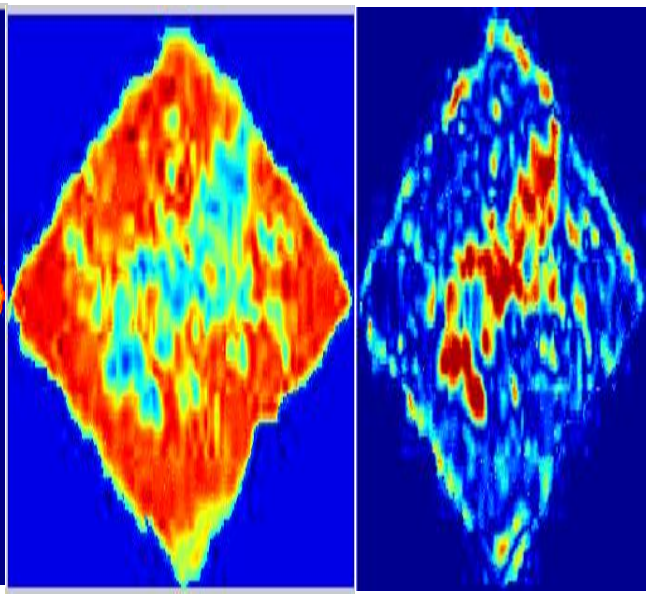

Figure (4) RGB image of a breast tissue sample depicting tumor traces. Score and residual image representations of the multivariate image upon performing MPCA decomposition

(a)

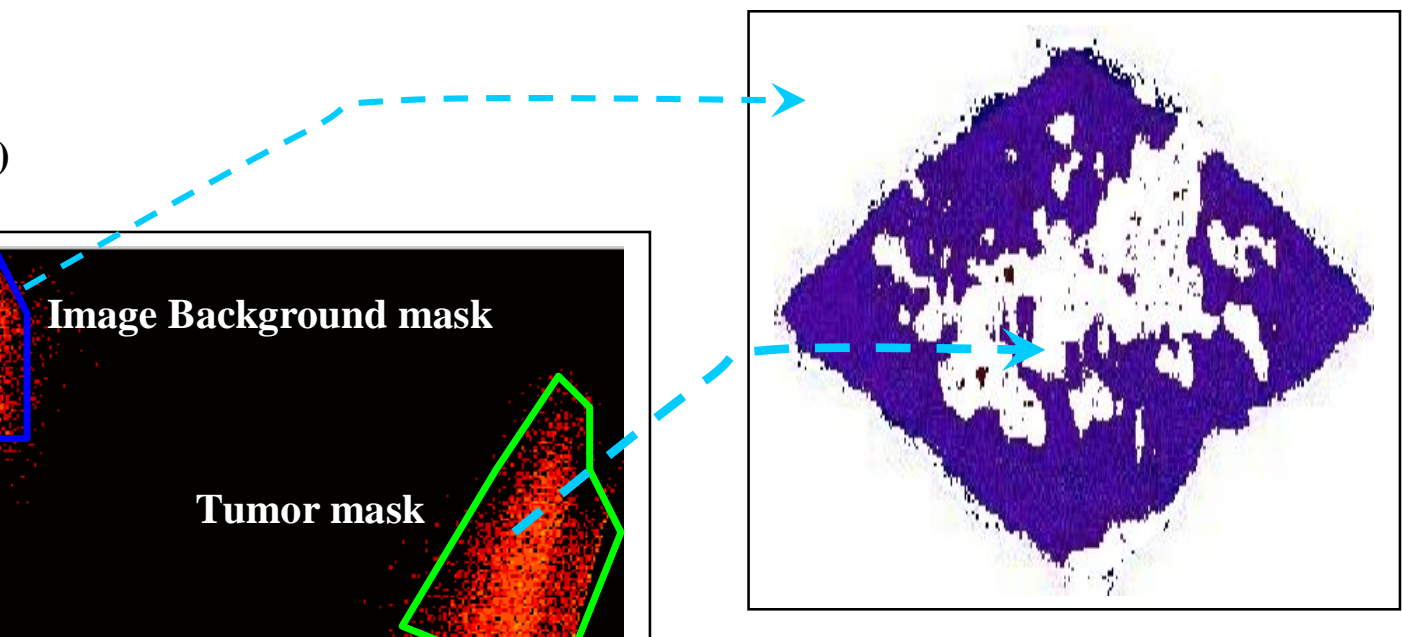

(b)
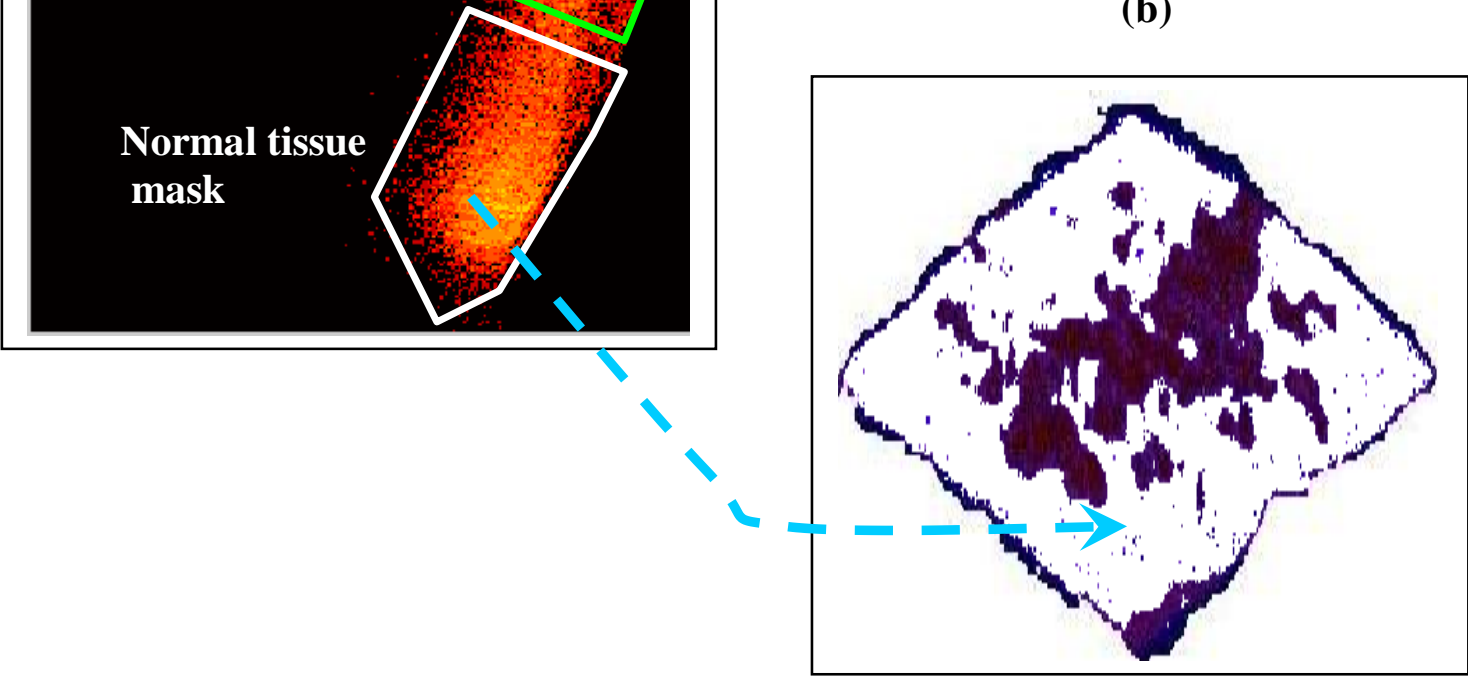
Figure 5. (a) t1-t2 score plot of tumor sample image with polygon masks. (b) False-color composite score image with overlay of highlighted pixels from the three classes outlined in part a.

A scatter plot of the first two score vectors $\left(t_{1} v t_{2}\right) s$ illustrated as a color coded two dimensional histogram in figure (5), where two main clusters exist, the top left represents the image background and the bottom right represents the image code. Also, $t_{1}$ values increase from right to left and $t_{2}$ values increase from top to bottom. Each point in this score plot represents a unique pixel in the image plane of the RGB multivariate image. Similar feature pixels in the original image yield almost identical $\left(t_{1}, t_{2}\right)$ score combinations, which results in many overlapping points in this scatter plots.

The number of overlapping pixels represented by a single point in a score plot is called the pixel density. A two dimensional histogram of $256 \times 256$ bins is constructed [28], where each bin is assigned a color depending on the number of pixels falling in that bin.

It uses black to represent bins containing no pixels and light colors to represent bins having the highest pixel density. The color coding progresses from black to white in various shades of red orange and yellow [28].

It is easy to detect outlier pixel clusters, as mentioned before, to the top-left and to the right of the score plot in figure (5) that are remote from the main pixel cluster in the bottom right. Pixels having similar spectral features in the multivariate image will have comparable combinations of score values and result in point clusters in the score plot.

The backbone of the MIA strategy is the use of masking [25] to delineate clusters of interest and to map pixels falling within these masks back into the image space to determine the features to which they correspond. In figure (5a), two polygon masks (blue and green) are placed around the two minor point clusters toward the top and right, respectively. The pixel classes that have been masked in this score plot are highlighted in figure (5b), where each pixel with $a t_{1}-t_{2}$ score combination lying under the respective masks has been replotted as an overlaid white pixel on a false color composite RGB image of the two PCs and residual ( $T_{1}=$ red, $T_{2}=$ green, $\left.E=b l u e\right)$.

From this figure, it is evident that the class of pixels highlighted by the green mask in the score space belongs to tumor traces, whereas the image background class is highlighted as the blue mask. By repeated use of the masking/ highlighting procedure with different mask sizes, a signature of feature existing in the image space can be isolated in the score space.

The above analysis can be easily implemented as a monitoring system to detect the breast cancer tumor. By using the local areas masks in figure (5), along with the loading vectors $\mathrm{p} 1$ and $\mathrm{p} 2$, a training model could then be used to detect the tumor existence from RGB images. Any new image sample must be rearranged into two way array and multiplied with the model loading vectors to produce the corresponding score vectors. The second step is to project the new score vectors back into the trained scatter plot, then check for the amount of feature pixels, along with pixels locations.

\section{5- Conclusions}

The optical generation and deflection of broadband surface acoustic wave pulses have been extended into the $100 \mathrm{MHz}$ region in the present experiment. Such a bandwidth is sufficient for the investigation of large number of biomedical application. The detection of SAW pulses has been improved considerably reaching limit of $0.2 \mathrm{~A}$ with interferometric detection scheme. This corresponds to the depth resolution of the scanning tunneling 
microscope. The lateral resolution is in the $\mu \mathrm{m} 2$ region and given by the focal width of the $\mathrm{HeNe}$ laser. The laser excitation of the SAWs is efficient enough that in most tissues the transient acoustic wave pulses can be monitored for laser pulse energies below the threshold for ablation in the thermoelastic regime. Thus, the technique is not only noncontact but also non-distractive.

The information is extractives from the shape of the acoustic wave pulse and the propagation of the transient acoustic pulse in the surface. In most tissues a broadening of acoustic wave pulse by the inherent structural properties is observed. The SAW amplitude yields important information on the optical properties (e.g., absorption coefficient) and physical properties (e.g., thermal expansion) of the sample. The measurement of the dispersion phase velocity in sample, which may perform with accuracy of several $\mathrm{m} / \mathrm{s}$ allows characterization of the sample by its elastic properties.

In time-resolved photoacoustic imaging, thermoelastic waves are generated by absorption of short laser pulses. The abrupt thermal expansion in the target produces an ultrasonic wave that's temporal and amplitude characteristics depend on the optical and acoustic properties of the tissue. A thermoelastic waves represents the photoacoustic signature of the target tissue. Time-resolved photoacoustic imaging suited to the detection and assessment malignant.

Measurements will first be made to validate experimental work that has identified specific differences in the optical properties of cancers compared to normal healthy tissues, and determine the wavelengths at which the differences in the photoacoustic and photothermal signatures are likely to be greatest. The thermal properties of these same tissues will be also measured since differences in the thermal diffusivity are affected on the photothermal response.

The designed optical sensors have very great diagnostic power since it would be able to measure simultaneously the optical, thermal, and acoustic properties of the tissue. It would also be able to measure the thickness of tissue layers.

Multivariate image analysis for the off-line detection of breast cancer have been presented and applied to a $150 \mu \mathrm{m}$ human breast tissues sample. The technique provides a qualitative result that can be used for early tumor detection. The proposed technique can potentially be used on-line to prescreen the existence of tumors through vision based systems.

\section{6- References}

1. Yasser H. El-Sharkawy, Yehia Badr, "Laser-induced photoacoustic imaging for characterizing biological tissues" Proceedings of SPIE -- Volume 5689 April 2005, pp. 165173

2. El-Sharkawy, Yasser H.; Badr, Y.; "Diagnostic of human teeth using photoacoustic response" Lasers in Dentistry XII. Edited by Rechmann, Peter; Fried, Daniel. Proceedings of the SPIE, Volume 6137, pp. 1-9 (2006).

3. Lahmann W, Ludewig HJ, \& Welling H (1977) "Opto-acoustic trace analysis in liquids with the frequency-modulated beam of an Argon ion laser". Analytical Chemistry 49: 549551.

4. Oda S, Sawada T \& Kamada H (1978) "Determination of ultra trace Cadmium by laserinduced photoacoustic absorption spectroscopy". Analytical Chemistry 50: 865- 867. 
5. Oda S, Sawada T, Nomura M \& Kamada H (1979) "Simultaneous determination of mixtures in liquid by laser-induced photoacoustic spectroscopy". Analytical Chemistry 15: 686-690.

6. G.J. Diebold, T. Sun, and M.I. Khan "Photoacoustic waveforms generated by fluid bodies" Springer Scrics in Optical Sciences, Vol.69, pp.263-269, 1992

7. J. G. Laufer, P. C. Beard, and T. N. Mills "Comparison of the photothermal ensitivity of an interferometric optical fiber probe with pulsed photothermal radiometry" Review of Scientific Instruments, Vol. 73, No. 9,2002

8. Albagli D, Dark M,von Rosenberg C, Perelman L T, Itzkan I and feld M S (1994b) "laser-induced thermoelastic deformation a three dimensional solution and its application to the ablation of biological tissue", Med.phys.21 1323-31

9. Albagli D, Dark M,von Rosenberg C, Perelman L T, Itzkan I and feld M S (1994b) "laser-induced thermoelastic deformation a three dimensional solution and its application to the ablation of biological tissue", Med.phys.21 1323-31

10. S. Siano, R. Pini, R. Salimbeni \& M. Vannini "A Diagnostic Set-up for Time- resolved Imaging of Laser-induced Ablation" Optics and Losers in Engineering 25 (1996) 1-12

11. George H. Pettit "laser in medicine" 2003.

12. Abraham Katzir "Laser and optical fibers in medicine" 1993.

$13 \mathrm{G}$. J. Diebold, Theory of thin layer photoacoustic cells for determination of volume changes in solution, J. Phys. Chem. B, 1998, 102, 5404-5408.

14. Marta L Dark, Lev T, Irving Itzkan, Jonaathan L and Michael S (1999)," physical properties of hydrated tissue determined by surface interferometry of laser-induced thermoelastic deformation". Phys. Med. Biol.45 (2000) 529-539

15. Albagli D, Dark M,von Rosenberg C, Perelman L T, Itzkan I and feld M S (1994b) "laser-induced thermoelastic deformation a three dimensional solution and its application to the ablation of biological tissue", Med.phys.21 1323-31

16. P.C. Beard, F.perennes, E. Draguioti, and T.N. (1998)," Mills Optical fiber photoacoustic-photothermal probe", Optical Society of America.

17. American Cancer Society. Cancer facts and figures. Technical report; 1991.

18. Tim W. Nattkemper, (2004), "Multivariate Image Analysis in Biomedicine-A Methodological Review", Journal of Biomedical Informatics.

19. L. Eriksson, E. Johansson, N. Kettaneh-Wold, S. Wold, Introduction to Multi- and Megavariate Data Analysis using Projection Methods (PCA and PLS), Umetrics, Umea ${ }^{\circ}$, Sweden, 1999.

20. P. Geladi, H. Grahn, Multivariate Image Analysis, John Wiley \& Sons, Baffins Lane, Chichester, 1996.

21. H. Yu, Development of Vision-based Inferential Sensors for Process Monitoring and Control, PhD thesis, McMaster University, Hamilton, Canada (2003).

22. M. Bharati, Multivariate Image Analysis and Regression Techniques for Industrial Process Monitoring and Product Quality Control, PhD thesis, McMaster University, Hamilton, Canada (2002).

23. M. Bharati, J.F. MacGregor, W. Tropper, (2003),"Softwood lumber grading through on-line multivariate image analysis techniques", Ind. Eng. Chem., 42, 5345-5353.

24. H. Yu, J. F. MacGregor, (2003), "Multivariate image analysis and regression for prediction of coating content and distribution in the production of snack foods", Chemometrics and Intelligent Laboratory Systems 67, 125-144.

25. M. Bharati, J.F. MacGregor, (1998), " Multivariate image analysis for real time process monitoring and control", Ind. Eng. Chem. Res., 37, 4715-4724.

26. K. Esbensen, P. Geladi, H. Grahn, Chemometrics and Intelligent Laboratory Systems 14 (1992) 67- 86. 
27. K. G. Dunn, (2007), "MACC Multivariate Image Analysis", McMaster Advanced Control Consortium (MACC), McMaster University, Ontario, Canada, http://macc.mcmaster.ca/

28. M. Bharati, (1997), " Multivariate image analysis for real time process monitoring and control", M. Eng. Thesis, McMaster Univeersity, Hamilton, ON, Canada. 